\title{
Teores de chumbo e mercúrio em cabelo de crianças residentes em Cubatão, na região sudeste do Brasil
}

\author{
Lead and mercury in the hair of children living in Cubatão in the Southeast \\ Region of Brazil
}

\author{
Eladio Santos Filho*, Rebeca de Souza e Silva"*, Alice M. Sakuma***, Maria Anita Scorsafava***
}

\begin{abstract}
SANTOS FILHO, E. et al. Teores de chumbo e mercúrio em cabelo de crianças residentes em Cubatão, na região sudeste do Brasil. Rev. Saúde Pública, 27:81-86, 1993. Realizou-se estudo de tipo seccional onde concentrações de chumbo e mercúrio foram determinadas pela técnica de espectrofotometria de absorção atômica em cabelo de 251 crianças de um a 10 anos de idade, residentes em seis bairros situados às margens dos principais rios do Município de Cubatão, SP, (Brasil). Verificou-se concentrações de chumbo em cabelo de 229 crianças, sendo que em 189 delas $(82,5 \%)$ detectou-se teores que variaram de 2,5 a $71,4 \mu \mathrm{g} / \mathrm{g}$. O teor médio de chumbo foi de $7,25 \pm$ $8,51 \mu \mathrm{g} / \mathrm{g}$. Verificou-se concentrações de mercúrio em cabelo de 217 crianças e em todas foram detectados teores que variaram de 0,2 a $3,0 \mu \mathrm{g} / \mathrm{g}$. A concentração média de mercúrio foi de $0,82 \pm$ $0,48 \mu \mathrm{g} / \mathrm{g}$. Não foi observada diferença estatística $(\mathrm{p}>0,05)$ dos teores médios de chumbo e mercúrio em cabelo entre crianças consumidoras de peixes e/ou produtos dos rios de Cubatão e crianças não-consumidoras de organismos aquáticos de qualquer origem.
\end{abstract}

Descritores: Chumbo, análise. Mercúrio, análise. Cabelo, química. Espectrofotometria por absorção atômica, utilização.

\section{Introduçáo}

O Município de Cubatão localiza-se no litoral sul do Estado de São Paulo, Brasil, a uma distância aproximada de $60 \mathrm{Km}$ da Capital do Estado. Ocupa uma área de $162 \mathrm{Km}^{2}$, onde abriga 23 indústrias do ramo petroquímico, siderúrgico, de refino de petróleo, de cimento, de fertilizantes e de metais não-ferrosos.

A partir do ano de 1980, o Município adquiriu fama internacional, devido a grande quantidade de poluentes químicos lançados sem controle no meio ambiente, prejudicando o ecossistema local. Em 1984, a Companhia de Tecnologia de Saneamento Ambiental (CETESB) implantou, no $\mathrm{Mu}$ nicípio, o Programa de Controle Ambiental ${ }^{5}$ com o objetivo de reduzir ou eliminar as 320 princi-

\footnotetext{
* Secretaria de Estado da Saúde de São Paulo - Cubatão, SP - Brasil

* Doutoranda da Faculdade de Saúde Pública da Universidade de São Paulo, SP - Brasil

*** Instituto Adolfo Lutz - São Paulo, SP - Brasil
}

Separatas/Reprints: E. Santos Filho - Rua Praia dos Sonhos, 79 - Vila Guilhermina - 11701-390.

Praia Grande, SP - Brasil.

Edição subvencionada pela FAPESP. Processo Medicina 93/ 0208-5 pais fontes de poluição identificadas, sendo 230 fontes de poluição do ar, 46 de poluição do solo e 44 de poluição das águas. Até o ano de implantação desse Programa, as 44 fontes de poluição das águas emitiam $64 \mathrm{t}$./dia de poluentes, dos quais reduziu-se, até o momento, $93,8 \% 7$. Em conseqüência desse controle, houve retorno dos peixes aos rios de Cubatão a partir do ano de 1988. Desde então os peixes e outros organismos da biota aquática do Município vêm sendo consumidos, principalmente pela população de menor nível socioeconômico.

Mais recentemente, estudo de contaminação da biota aquática da bacia do rio Cubatão, realizado pela CETESB ${ }^{6}$, verificou que os peixes e outros organismos aquáticos estão impróprios ao consumo humano devido à presença de altos teores de chumbo com cerca de $50 \%$ das amostras de musculatura (parte comestível), apresentando teores acima dos limites permissíveis de $0,5 \mu \mathrm{g} / \mathrm{g}$. $O$ mercúrio foi encontrado em $5,6 \%$ das amostras de musculatura dos peixes em níveis superiores aos valores permissíveis de $0,5 \mu \mathrm{g} / \mathrm{g} .{ }^{6} \mathrm{O}$ maior teor de mercúrio encontrado nos peixes foi de $0,9 \mu \mathrm{g} / \mathrm{g}$.

Dentre os vários tipos de amostras biológicas, o cabelo tem sido reconhecido como acumulador de metais pesados em suas fibras e pode ser usado como um excelente indicador de envenenamento agudo por esses metais ${ }^{1,9,15}$. 
Em função disso e aliado ao fato de que a população residente em determinados bairros do $\mathrm{Mu}$ nicípio de Cubatão, notadamente a de menor ren$\mathrm{da}$, vem consumindo largamente peixes e outros produtos contaminados dos rios do Municipio, e ainda devido à inexistência de trabalhos com o objetivo de verificar as concentraçōes de xenobióticos em habitantes da região, decidiu-se realizar pesquisa nessa linha.

O objetivo do presente trabalho é avaliar os níveis de concentração de chumbo e mercúrio em cabelo de crianças residentes em bairros situados às margens dos rios de Cubatão.

\section{Material e Método}

\section{Delineamento do estudo}

Para eliminar as variáveis de confundimento como o tabagismo e a exposição ocupacional, decidiu-se realizar a pesquisa com crianças de 1 a 10 anos de idade. Para a determinação dos teores capilares dos xenobióticos estudados, foi realizado inquérito do tipo transversal. Para estabelecer relação entre os teores capilares encontrados e o consumo de peixes e/ou outros organismos dos rios de Cubatão, foram formados dois grupos constituídos de crianças, grandes consumidoras e não-consumidoras, respectivamente. Foram consideradas grandes consumidoras as crianças com referência de consumo de peixes e/ou outros produtos dos rios de Cubatão com a frequiência maior ou igual a uma vez por semana e por um período nunca inferior a 6 meses. No grupo de nãoconsumidoras foram incluídas as crianças com referência negativa de consumo de peixes e/ou outros produtos aquáticos de qualquer origem.

\section{Local do estudo}

A pesquisa foi realizada em seis bairros do $\mathrm{Mu}-$ nicípio de Cubatão, escolhidos por apresentarem característica comum de se localizarem às margens dos rios e com facilidade de acesso a eles. As características dos bairros pesquisados foram apresentadas na primeira parte deste estudo ${ }^{19}$.

\section{Instrumento de medida}

Para a determinação da frequiência de consumo dos produtos dos rios, elaborou-se um formulário de inquérito alimentar, utilizando-se o método recordatório dos últimos seis meses da data de aplicação do formulário. Acredita-se que este periodo de tempo não tenha prejudicado a memória dos entrevistados devido a alta especificidade do alimen- to pesquisado. A aferição do instrumento de medida foi feita através de pré-teste em 57 familias residentes nos bairros pesquisados mas não pertencentes à amostra. A aplicação do formulário foi feita por duas estudantes de nível secundário, especialmente treinadas.

\section{Seleção das unidades amostrais}

Os recursos disponíveis para a realização da presente pesquisa foram determinantes na decisão de se trabalhar com uma amostra de fácil acesso, não probabilística. Nessa eventualidade, os resultados obtidos refletem, unicamente, a situação das crianças pesquisadas, isto $\epsilon$, não devem ser generalizados para a população alvo.

Em definitivo, foram visitadas 773 residências, sendo que 155 foram consideradas fechadas após duas tentativas em dias diferentes e em 7 residências houve recusa a entrevista. Nas 611 residências que se teve acesso, existiam 696 crianças de 1 a 10 anos de idade. Excluídas as crianças que não se enquadraram como grandes consumidoras ou nãoconsumidoras, restaram 346 que foram convidadas, através visita domiciliar, a comparecer em local e data pré-determinados, para coleta de material para exames laboratoriais. Responderam ao convite 281 crianças $(81,2 \%)$. Durante a coleta de material, reformularam-se as questões do inquérito alimentar e, assim, excluiu-se da amostra 30 crianças que não confirmaram informaçōes anteriores sobre consumo de peixes e/ou outros produtos aquáticos. $\mathrm{O}$ universo da pesquisa constituiuse, então de 251 crianças, divididas em 53 grandes consumidoras de peixes e/ou outros organismos dos rios de Cubatão mas também de outras origens $(++), 18$ grandes consumidoras de organismos aquáticos exclusivamente dos rios de Cubatão (+-), 148 grandes consumidoras de peixes e/ou outros produtos de diversas origens que não os rios de Cubatão (-+), e 32 crianças não-consumidoras de organismos aquáticos de qualquer origem (--). Para estabelecer relação entre concentração capilar dos xenobióticos estudados e consumo de peixes dos rios de Cubatão, utilizou-se os dados apenas das crianças classificadas como +- e -..

\section{Coleta de material}

A equipe de coleta de material foi composta por um médico pediatra, uma enfermeira de nivel universitário e dois auxiliares de enfermagem. As crianças compareceram nos postos de coleta, 10calizados invariavelmente nos postos de saúde ou escolas dos bairros, acompanhadas dos pais ou responsáveis. Foram submetidas a um exame físico e a um breve interrogatório sobre o uso de cosméti- 
cos, medicamentos tópicos ou sistêmicos e questões do inquérito alimentar. Após tais procedimentos, eram colhidos cerca de $300 \mathrm{mg}$ de cabelo cortados rentes ao couro cabeludo, e ao final de cada jornada de trabalho todo o material era imediatamente entregue ao Instituto Adolfo Lutz, responsável pela execução das análises.

\section{Metodologia laboratorial}

As amostras de cabelo foram previamente lava$\operatorname{das}^{14}$ e homogeneizadas. O mercúrio foi analisado por espectrofotometria de absorção atômica sem chama ${ }^{3}$. Para a determinação do chumbo, as amostras de cabelo foram mineralizadas com ácido nítrico e peridrol. $O$ resíduo foi dissolvido com ácido clorídrico e o chumbo ex traído com APDC/MIBC ${ }^{1} \mathrm{e}$ determinados por espectrofotometria de absorção atômica. Para efeito dos cálculos estatísticos, os resultados inferiores ao limite de detecção do método empregado foram considerados iguais a zero.

\section{Análise estatística dos dados}

Para avaliar se as concentraçð̃es médias de xenobióticos dos diversos subgrupos diferiam significativamente entre si, recorreu-se à análise de variância (ANOVA). Em uma primeira instância, mediante a utilização do teste de Bartlett's, verificou-se se as variâncias dos diferentes grupos poderiam ser consideradas homogêneas. Em caso afirmativo, os resultados da ANOVA foram utilizados. Em caso negativo, recorreu-se ao teste de Kruskal-Wallis, não paramétrico, para tomada de decisão. Trabalhou-se com o nivel usual de $5 \%$ de significância.

\section{Resultados e Discussão}

Das 251 crianças da amostra, analisou-se o chumbo no cabelo de 229 crianças, sendo que em 189 delas $(82,5 \%)$ detectou-se concentraçōes que variaram de 2,5 a $71,4 \mu \mathrm{g} / \mathrm{g}$. A Tabela 1 mostra a concentração média de chumbo em cabelo, observando-se que o teor médio encontrado foi de 7,25 $\pm 8,51 \mu \mathrm{g} / \mathrm{g}$.

Pueschel e col. ${ }^{17}$, em estudo de 343 crianças norte-americanas, encontraram a concentração média de $0,37 \pm 0,15 \mu \mathrm{g} / \mathrm{g}$ de chumbo em cabelo. Shrestha e Schrauzer ${ }^{21}$ encontraram em adultos não expostos ao chumbo os teores capilares de 8,5 $\pm 5,6 \mu \mathrm{g} / \mathrm{g}$ em Darjeeling (India), e de 6,3 $\pm 8,1 \mu \mathrm{g} /$ $\mathrm{g}$ em San Diego (USA). Salgado e Lepera ${ }^{18} \mathrm{em}$ estudo preliminar de 34 crianças de 7 a 10 anos, encontraram a concentração média de $7,4 \pm 3,8 \mu \mathrm{g} / \mathrm{g}$ de chumbo em cabelo.
Ainda na Tabela 1, verifica-se que a concentração média mais elevada ocorreu na faixa etária de 1 a 2 anos de idade, embora não se tenha encontrado diferença estatística comparando-se com as médias das demais faixas etárias.

Eltayeb e Grieken ${ }^{8}$ ao estudarem os teores médios de metais em cabelo de diversos grupos etários, encontraram diferença estatística entre as médias aritméticas de chumbo em cabelo de crianças e adolescentes sudaneses $(10,3 \pm 5,8 \mu \mathrm{g} /$ g) e de pessoas idosas $(3,4 \pm 2,9 \mu \mathrm{g} / \mathrm{g})$. Carvalho e col. ${ }^{4}$ verificaram em crianças do interior da Bahia, que os teores de chumbo em cabelo diminuíam proporcionalmente em relação ao aumento da idade. A mesma relação inversa foi achada por Shuhmacher e col. ${ }^{22}$.

Tabela 1. Concentraçōes médias de chumbo em cabelo por faixa etária de crianças residentes em Cubatão, SP.

\begin{tabular}{cccc}
\hline Faixa etária & $N$ & $\begin{array}{c}\text { Var. Individuais } \\
\mu g / g\end{array}$ & $\begin{array}{c}\text { Média } \pm \mathrm{DP} \\
\mu \mathrm{g} / \mathrm{g}\end{array}$ \\
\hline $1 \vdash 3$ & 37 & $0 \longleftrightarrow-71,4$ & $9,82 \pm 13,42$ \\
$3-5$ & 51 & $0 \longleftrightarrow 32,8$ & $6,92 \pm 6,31$ \\
$5-7$ & 49 & $0 \longleftrightarrow 29,7$ & $6,45 \pm 6,56$ \\
$7-9$ & 43 & $0 \longleftrightarrow 40,8$ & $6,78 \pm 7,64$ \\
$91-11$ & 49 & $0 \longleftrightarrow 43,7$ & $6,93 \pm 8,54$ \\
\hline Total & 229 & $0 \longleftrightarrow 71,4$ & $7,25 \pm 8,51$ \\
\hline
\end{tabular}

A Tabela 2 mostra que o teor médio de chumbo em cabelo foi significativamente menor no sexo masculino, comparado ao sexo feminino. Atribuiuse, no entanto, este achado ao tempo do corte de cabelo ter sido maior nas meninas (mediana $=90$ dias) em relação aos meninos (mediana $=30$ dias), do que propriamente uma diferença ligada ao sexo. Reforça este argumento, o fato de não ter sido encontrada diferença significativa entre as concentrações sanguíneas do chumbo por sexo, como já publicado na primeira parte deste trabalho ${ }^{19}$.

Bergomi e col. ${ }^{1}$, pesquisando 111 escolares italianos residentes em área industrial, verificaram a concentração média de $25,11 \pm 26,79 \mu \mathrm{g} / \mathrm{g}$ de chumbo em cabelo. $O$ sexo masculino apresentou teor médio de $29,31 \pm 28,8 \mu \mathrm{g} / \mathrm{g}$ e o feminino o teor

Tabela 2. Concentrações médias de chumbo em cabelo por sexo em crianças residentes em Cubatão, SP.

\begin{tabular}{lccc}
\hline Sexo & $N$ & $\begin{array}{c}\text { Var. Individuais } \\
\mu \mathrm{g} / \mathrm{g}\end{array}$ & $\begin{array}{c}\text { Média } \pm \mathrm{DP} \\
\mu \mathrm{g} / \mathrm{g}\end{array}$ \\
\hline Masculino & 105 & $0 \longleftrightarrow \longrightarrow 71,4$ & $6,03 \pm 8,49$ \\
Feminino & 124 & $0 \longleftrightarrow 43,7$ & $8,27 \pm 8,43$ \\
\hline Total & 229 & $0 \longleftrightarrow 71,4$ & $7,25 \pm 8,51$ \\
\hline
\end{tabular}


médio de $21,55 \pm 24,55 \mu \mathrm{g} / \mathrm{g}$. Leotsinidis e Kondakis $^{10}$, no final da década de 80 , ao estudarem os teores de metais em cabelo de agricultores vivendo em área não poluída na Grécia, não encontraram diferença estatística entre as concentraçōes médias de chumbo no sexo masculino $(4,40 \pm 1,97 \mu \mathrm{g} / \mathrm{g})$ e no sexo feminino $(3,84 \pm 2,08 \mu \mathrm{g} / \mathrm{g})$.

A Tabela 3 mostra as concentraç̃es médias de chumbo em cabelo, por bairro. Verificou-se que o bairro "Caminho dos Pilões" apresentou a média mais elevada de chumbo em cabelo, cerca de duas vezes superior aos demais bairros pesquisados. Em janeiro de 1991 foi descoberta a existência de depósitos clandestinos de resíduos sólidos industriais no Vale dos Pilōes, a uma distância aproximada de $3 \mathrm{Km}$ a montante deste núcleo habitacional. A CETESB constatou, dentre outros poluentes, a presença de concentrações elevadas de chumbo (até $63,8 \mathrm{mg}$ / $\mathrm{kg}$ ), o que poderia explicar os teores mais elevados de chumbo em cabelo nesse bairro. Menzer ${ }^{11}$ descreve que a presença de chumbo no solo se encontra, geralmente, no teor médio de $10 \mathrm{a} 15 \mathrm{mg} / \mathrm{kg}$.

Tabela 3. Concentrações médias de chumbo em cabelo por local de residência, em crianças, Cubatão, SP.

\begin{tabular}{|c|c|c|c|}
\hline Bairro & $N$ & $\begin{array}{c}\text { Var. Individuais } \\
\mu \mathrm{g} / \mathrm{g}\end{array}$ & $\begin{array}{c}\text { Média } \pm \text { DP } \\
\mu g / g\end{array}$ \\
\hline $\begin{array}{l}\text { Jd. Costa e Silva } \\
\text { Vila Elizabeth } \\
\text { Vila Pescadores } \\
\text { Pelicas } \\
\text { Caminho dos Trilhos } \\
\text { Camiriho dos Pilōes }\end{array}$ & $\begin{array}{r}72 \\
56 \\
53 \\
9 \\
17 \\
22\end{array}$ & $\begin{array}{l}0 \longleftrightarrow-40,8 \\
0 \longleftrightarrow-35,0 \\
0 \longleftrightarrow->71,4 \\
0 \longleftrightarrow-14,3 \\
0 \longleftrightarrow-19,5 \\
0 \longleftrightarrow->43,7\end{array}$ & $\begin{array}{c}6,74 \pm 7,78 \\
7,24 \pm 7,14 \\
6,82 \pm 10,51 \\
5,84 \pm 5,72 \\
5,11 \pm 5,70 \\
12,15 \pm 10,36\end{array}$ \\
\hline Total & $229^{\circ}$ & $0 \longleftrightarrow-71,4$ & $7,25 \pm 8,51$ \\
\hline
\end{tabular}

Na Tabela 4 vemos a concentração média de chumbo em cabelo por situação de consumo de peixes e/ou outros organismos dos rios de $\mathrm{Cu}-$ batão. Não foi observada diferença estatística entre as médias encontradas.

A Tabela 5 mostra as concentrações médias de mercúrio em cabelo por faixa etária. Não foi observada diferença estatística entre os teores médios apresentados.

Turner e col. ${ }^{24}$ verificaram entre 128 habitantes da cidade de Morropon, no Peru, teor médio de

Tabela 4. Concentração média de chumbo em cabelo por situação de consumo em crianças residentes em Cubatão, SP.

\begin{tabular}{lccc}
\hline Consumo & $N$ & $\begin{array}{c}\text { Var. Individuais } \\
\mu \mathrm{g} / \mathrm{g}\end{array}$ & $\begin{array}{c}\text { Média } \pm \mathrm{DP} \\
\mu \mathrm{g} / \mathrm{g}\end{array}$ \\
\hline $\begin{array}{l}\text { Grande consumidor } \\
\text { Não-consumidor }\end{array}$ & 14 & $0 \longleftrightarrow->19,5$ & $7,52 \pm 5,78$ \\
\hline
\end{tabular}

Tabela 5. Concentrações médias de mercúrio em cabelo por faixa etária em crianças residentes em Cubatão, SP.

\begin{tabular}{cccc}
\hline Faixa etária & $N$ & $\begin{array}{c}\text { Var. Individuais } \\
\mu \mathrm{g} / \mathrm{g}\end{array}$ & $\begin{array}{c}\text { Média } \pm \mathrm{DP} \\
\mu \mathrm{g} / \mathrm{g}\end{array}$ \\
\hline $1 \vdash 3$ & 34 & $0,3 \longleftrightarrow->1,4$ & $0,69 \pm 0,26$ \\
$3 \models 5$ & 48 & $0,2 \longleftrightarrow-3,0$ & $0,92 \pm 0,59$ \\
$5 \vdash 7$ & 47 & $0,3 \longleftrightarrow-2,9$ & $0,83 \pm 0,48$ \\
$7 \vdash 9$ & 42 & $0,2 \longleftrightarrow-2,7$ & $0,76 \pm 0,52$ \\
$9 \vdash 11$ & 46 & $0,3 \longleftrightarrow->2,5$ & $0,84 \pm 0,42$ \\
\hline Total & 217 & $0,2 \longleftrightarrow \longrightarrow 3,0$ & $0,82 \pm 0,48$ \\
\hline
\end{tabular}

$0,78 \mu \mathrm{g} / \mathrm{g}$ de mercúrio em cabelo. Sherlock e col. ${ }^{20}$ encontraram a concentração média de $1,4 \pm$ $2,4 \mu \mathrm{g} / \mathrm{g}$ de mercúrio em cabelo em 942 habitantes do Reino Unido.

Os teores médios de mercúrio em cabelo em população não diretamente exposta a este metal, encontrados por estes pesquisadores, são parecidos com o teor médio encontrado nas crianças pesquisadas em Cubatão, o que parece indicar a inexistência de exposição recente ao mercúrio.

A Organização Mundial de Saúde ${ }^{12}$ refere o aparecimento de sintomas de intoxicação pelo metilmercúrio em concentrações capilares variando de 50 a $120 \mu \mathrm{g} / \mathrm{g}$.

A Tabela 6 mostra a concentração média de mercúrio em cabelo por sexo. Não houve diferença estatisticamente significante entre os teores médios encontrados.

Wakisaka e col..$^{25}$ encontraram diferença significativa entre a concentração média capilar de mercúrio em homens adultos $(6,40 \pm 4,69 \mu \mathrm{g} / \mathrm{g})$ comparada à de suas esposas $(2,86 \pm 1,97 \mu \mathrm{g} / \mathrm{g})$. Oskarsson e col. ${ }^{13}$, pesquisando a concentração de mercúrio em cabelo de 50 pessoas, também verificaram que o teor médio no sexo masculino $(3,80 \pm$ $2,60 \mu \mathrm{g} / \mathrm{g}$ ) foi significativamente maior do que no sexo feminino $(2,40 \pm 1,80 \mu \mathrm{g} / \mathrm{g})$. Suzuki e col. ${ }^{23}$, à semelhança dos presentes resultados, não encontraram diferença estatística entre os teores médios de mercúrio em cabelo com relação ao sexo.

$\mathrm{Na}$ Tabela 7 são apresentadas as concentrações médias de mercúrio em cabelo por situação de consumo de peixes e/ou outros organismos aquáticos dos rios de Cubatão. Verifica-se que o teor médio de mercúrio $(0,98 \pm 0,44 \mu \mathrm{g} / \mathrm{g})$, em ca-

Tabela 6. Concentrações médias de mercúrio em cabelo por sexo, em crianças residentes em Cubatão, SP.

\begin{tabular}{lccc}
\hline Sexo & $\mathrm{N}$ & $\begin{array}{c}\text { Var. Individuais } \\
\mu \mathrm{g} / \mathrm{g}\end{array}$ & $\begin{array}{c}\text { Média } \pm \mathrm{DP} \\
\mu \mathrm{g} / \mathrm{g}\end{array}$ \\
\hline Masculino & 98 & $0,3 \longleftrightarrow->3,0$ & $0,83 \pm 0,47$ \\
Feminino & 119 & $0,2 \longleftrightarrow->2,7$ & $0,81 \pm 0,49$ \\
\hline Total & 217 & $0,2 \longleftrightarrow->3,0$ & $0,82 \pm 0,48$ \\
\hline
\end{tabular}


Tabela 7. Concentração média de mercúrio em cabelo por situação de consumo, em crianças residentes em Cubatão, SP.

\begin{tabular}{lccc}
\hline Consumo & $\mathrm{N}$ & $\begin{array}{c}\text { Var. Individuais } \\
\mu \mathrm{g} / \mathrm{g}\end{array}$ & $\begin{array}{c}\text { Média } \pm \mathrm{DP} \\
\mu \mathrm{g} / \mathrm{g}\end{array}$ \\
\hline Grande consumidor & 13 & $0,3 \longleftrightarrow \longrightarrow 1,7$ & $0,98 \pm 0,44$ \\
Não-consumidor & 24 & $0,3 \longleftrightarrow \longrightarrow 1,6$ & $0,70 \pm 0,31$ \\
\hline
\end{tabular}

belo das crianças consumidoras de produtos dos rios, não difere estatisticamente do teor médio $(0,70 \pm 0,31 \mu \mathrm{g} / \mathrm{g})$ apresentado pelas crianças nãoconsumidoras de organismos aquáticos de qualquer origem. Como já publicado na primeira parte deste trabalho ${ }^{19}$, a concentração média de mercúrio no sangue de crianças consumidoras de peixes e/ou outros produtos dos rios de Cubatão foi significativamente maior do que a concentração média de mercúrio no sangue de crianças não-consumidoras.

Oskarsson e col..$^{13}$ encontraram em adultos, grandes consumidores de peixes de água doce, o teor médio de $3,2 \pm 2,3 \mu \mathrm{g} / \mathrm{g}$ de mercúrio em cabelo. Birke ${ }^{2}$ encontrou em adultos não expostos ao mercúrio o teor capilar médio de $1,6 \mu \mathrm{g} / \mathrm{g}$. Em 12 adultos, grandes consumidores de peixes, encontrou a concentração média de $35,06 \mu \mathrm{g} / \mathrm{g}$. Phelps e col. ${ }^{16}$ verificaram entre indígenas canadenses, grandes consumidores de peixe contaminado com metilmercúrio, a concentração média de 13,8 $\pm 3,6$ $\mu \mathrm{g} / \mathrm{g}$ de mercúrio total em cabelo.

Tabela 8. Concentraçōes médias de mercúrio em cabelo por local de residência, em crianças de Cubatão, SP.

\begin{tabular}{|c|c|c|c|}
\hline Bairro & $\mathrm{N}$ & $\begin{array}{c}\text { Var. Individuais } \\
\mu \mathrm{g} / \mathrm{g}\end{array}$ & $\begin{array}{c}\text { Média } \pm D P \\
\mu \mathrm{g} / \mathrm{g}\end{array}$ \\
\hline $\begin{array}{l}\text { Jd. Costa e Silva } \\
\text { Vila Elizabeth } \\
\text { Vila Pescadores } \\
\text { Pelicas } \\
\text { Caminho dos Trilhos } \\
\text { Caminho dos Piloes }\end{array}$ & $\begin{array}{r}72 \\
60 \\
54 \\
9 \\
17 \\
5\end{array}$ & $\begin{array}{l}0,3 \longleftrightarrow 3,0 \\
0,3 \longleftrightarrow-7,7 \\
0,2 \longleftrightarrow-3,6 \\
0,3 \longleftrightarrow-1,5 \\
0,6 \longleftrightarrow-2,9 \\
0,4 \longleftrightarrow-1,6\end{array}$ & $\begin{array}{l}0,72 \pm 0,48 \\
0,79 \pm 0,32 \\
0,84 \pm 0,52 \\
0,81 \pm 0,34 \\
1,26 \pm 0,65 \\
0,80 \pm 0,47\end{array}$ \\
\hline Total & 217 & $0,2 \longleftrightarrow 3,0$ & $0,82 \pm 0,48$ \\
\hline
\end{tabular}

A Tabela 8 mostra as concentrações médias de mercúrio em cabelo por local de residência. Não se encontrou diferença estatística entre as médias apresentadas por cada bairro.

\section{Conclusó :s}

O teor médio de chumbo em cabelo foi de 7,25 $\pm 8,5 \mu \mathrm{g} / \mathrm{g}$. A maior média observada ocorreu na faixa de 1 a 2 anos de idade $(9,82 \pm 13,42 \mu \mathrm{g} / \mathrm{g})$, mas sem apresentar diferença estatisticamente significativa $(p>0,05)$ em comparação aos valores médios apresentados pelas demais faixas de idade. $\mathrm{O}$ teor médio mais elevado de chumbo em cabelo ocorreu no bairro Caminho dos Pilões $(12,15$ $\pm 10,36 \mu \mathrm{g} / \mathrm{g}$ ), quase duas vezes maior que os teores médios dos demais bairros.

A concentração média de mercúrio em cabelo foi de $0,82 \pm 0,48 \mu \mathrm{g} / \mathrm{g}$. Não se encontrou diferença estatisticamente significante $(p>0,05)$ entre os teores médios de mercúrio em cabelo de acordo com a faixa etária e com o local de residência. Não houve diferença estatística $(p>0,05)$ entre os teores médios de mercúrio em cabelo de crianças, grandes consumidoras de peixes e/ou outros produtos dos rios de Cubatão, e crianças nãoconsumidoras.

SANTOS FILHO, E. et al. [Lead and mercury in the hair of children living in Cubatão in the Southeast Region of Brazil]. Rev. Saúde Pública, 27: 81-86, 1993. A study was carried out in 6 districts of Cubatão county, $S$. Paulo State, Brazil on a sample of 251 children aged between 1 and 10. The concentration of heavy metals (lead and mercury) in hair was tested. The lead concentration in the hair of 229 children was tested, and in 189 (82.5\%) levels varying from 2.5 to $71.4 \mu \mathrm{g} / \mathrm{g}$ were detected. The average concentration of the lead found in hair was of $7.25 \pm 8.51 \mu \mathrm{g} / \mathrm{g}$. The concentration of mercury in the hair of 217 children was checked and all of them were found to have levels varying from 0.2 to 3.0 $\mu \mathrm{g} / \mathrm{g}$. The average concentration of mercury was of 0.82 $\pm 0.48 \mu \mathrm{g} / \mathrm{g}$. No statistical difference ( $p>0.05$ ) was observed between the averages found for lead and mercury in the hair of those who consumed and those who did not consume aquatic organisms.

Keywords: Lead, analysis. Mercury, analysis. Hair, chemical. Spectrophotometry, atomic absortion.

\section{Referências Bibliográficas}

1. BERGOMI, M. et al. Distribuzione del piombo nel sangue e nei capelli di una popolazione infantile residente in un'area industriale. Ann. Ist. Super Sanitá. 21: 43-52, 1985.

2. BIRKE, G. et al. Studies on humans exposed to methylmercury through fish consumption. Arch. Environ. Health, 25: $77.91,1972$.

3. CAMPE, A. et al. Determination of inorganic, phenyl and alkyl mercury in hair. Atom. Spectrosc., 3 (4): 122-5, 1982.

4. Carvalho, F. M. et al. Chumbo e cádmio em cabelo de crianças de Santo Amaro da Purificação, Bahia. Ciênc. e Cult., 41: 646-51, 1989.

5. COMPANHIA DE TECNOLOGIA DE SANEAMENTO AMBIENTAL (CETESB). Controle da poluição ambiental em Cubatão: resultados-julho/83 a janeiro/87. Sāo Paulo, 1987. 
6. COMPANHIA DE TECNOLOGIA DE SANEAMENTO AMBIENTAL (CETESB). Contaminantes na bacia do rio Cubatão e seus reflexos na biota aquática. São Pau. lo, 1990.

7. COMPANHIA DE TECNOLOGLA DE SANEAMENTO AMBIENTAL (CETESB). Ação da CETESB em Cubatão: situaçâo em janeiro de 1990. Sāo Paulo, 1990.

8. ELTAYEB, M. A. H. \& GRIEKEN, R. E. V. Iron, cooper, zinc and lead in hair from sudanese populations of different age groups. Sci Total Environ., 95: 157-65, 1990.

9. GREENWOOD, M. R. et al. Epidemiological experience with Mago's reagents in the determination of different forms of mercury in biological samples by flameless atomic absorption. J. Anal. Toxic., 1: 265-9, 1977.

10. LEOTSINIDIS, $M$. \& KONDAKIS, $X$. Trace metals in scalpe hair of Greek agricultural workers. Sci. Total Environ., 95: 149-56, 1990.

11. MENZER, R. E. Water and soil pollutants. In: Klaassen, C. D. et al. Casarett and Doull's Toxicology. 4a. ed. New York, Macmillan, 1991. p. 872-902.

12. ORGANIZAÇĀO MUNDIAL DA SAÚDE. Mercury. Geneva, 1976. (OMS-Environmental health criteria 1).

13. OSKARSSOM, A. et al. Mercury levels in hair from people eating large quantities of Swedish freshwater fish. Food Addit. Contam., 4: 555-62, 1990.

14. PETER, F. \& STRUNC, G. Semiautomated analysis for mercury in whole blood, urine and hair by on stream generation of cold vapor. Clin. Chem., 30: 893-5, 1984.

15. PETERNNG, H. G. et al. Trace metal content of hair. Arch. Environ. Health., 27: 327-30, 1973.

16. PHELPS, R. W. et al. Interrelationships of blood and hair mercury concentrations in a North American population exposed to methylmercury. Arch. Environ. Health., 35: 161-8, 1980.
17. PUESCHEL, S. M. et al. A screening and follow up study of children with a increased lead burdens. JAMA, 222: 462-6, 1972

18. SALGADO, P. E. T. \& LEPERA, J. S. Teores de chumbo, zinco e cobre em amostras de cabelo de crianças: estudos preliminares. Rev. Ciênc. Farm., 7: 61-5, 1985.

19. SANTOS FILHO, E. et al. Concentraçöes sangüíneas de metais pesados e praguicidas organoclorados em crianças de 1 a 10 anos. Rev. Saúde Pública, 27: 59-68, 1993.

20. SHERLOCK, J. C. et al. Duplication diet study on mercury intake by fish consummers in the United Kingdom. Arch. Environ. Health, 37: 271-3, 1982.

21. SHRESTHA, K. P. \& SCHRAUZER, G. N. Trace elements in hair: a study of residents in Darjeeling (India) and San Diego, Califomia (USA). Sci. Total Environ., 79: 171-7, 1989.

22. SHUHMACHER, M. et al. Lead in children's hair, as related to exposure in Tarragona Province, Spain. Sci. Totatl Environ., 104: 167.73, 1991.

23. SUSUKI, T. et al. Comparison of hair with nail as index media for biological monitoring of mercury. Sangyo Jgakn., 31: 235-8, 1989.

24. TURNER, M. D. et al. Methylmercury in populations cating large quantities of marine fish. Arch. Environ. Health, 35: 367-78, 1980.

25. WAKISAKA, I. et al. Factors contributing to the difference of hair mercury concentrations between the sexes. Nip. pon Eisei. Zasshi, 45: 654-64, 1990.

Recebido para publicação em 5.3.1992

Reapresentado em 10.12.1992

Aprovado para publicação em 21.12.1992 